\title{
Design and synthesis of multivalent neoglycoconjugates by click conjugations
}

Feiqing Ding, Li Ji, Ronny William, Hua Chai and Xue-Wei Liu*

\section{Full Research Paper}

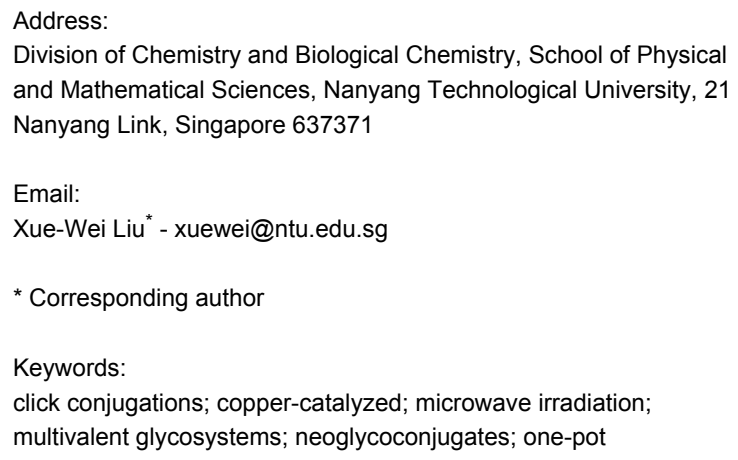

Open Access
doilstein J. Org. Chem. 2014, 10, 1325-1332.
Received: 24 February 2014
Accepted: 21 May 2014
Published: 10 June 2014
This article is part of the Thematic Series "Multivalent glycosystems for
nanoscience".
Guest Editor: B. Turnbull
(c) 2014 Ding et al; licensee Beilstein-Institut.
License and terms: see end of document.

\begin{abstract}
A highly stereoselective $\mathrm{BF}_{3} \cdot \mathrm{OEt}_{2}$-promoted tandem hydroamination/glycosylation on glycal scaffolds has been developed to form propargyl 3-tosylamino-2,3-dideoxysugars in a one-pot manner. Subsequent construction of multivalent 3-tosylamino-2,3-dideoxyneoglycoconjugates with potential biochemical applications was presented herein involving click conjugations as the key reaction step. The copper-catalyzed regioselective click reaction was tremendously accelerated with assistance of microwave irradiation.
\end{abstract}

\section{Introduction}

Oligosaccharides and glycopeptides are the key constituents of the cellular membrane and extracellular matrix, and play a pivotal role in various key cellular events such as cell-cell recognition, host-pathogen or host-symbiont interactions, molecular recognition of antibodies and metastasis [1-5]. The construction of a 1,4-disubstituted-1,2,3-triazole unit via a copper(I)-catalyzed modern version of the Huisgen-type azide-alkyne cycloaddition [6-10] has been considered to be a powerful ligation method for glycoconjugation [11-16]. In addition to the simplicity of this reaction and the ease of purification, 1,4-disubstituted-1,2,3-triazoles, the regiospecific product of this reaction, exhibit similarities to the ubiquitous amide moiety found in nature. However, unlike amides, the triazole moiety proved to be robust and resistant to chemical and enzymatic cleavage [17-20]. Moreover, the inertness of both azide and alkyne groups towards a majority of functional groups connected to the core of a variety of biomolecules also renders the click reaction particularly suitable for covalently linking bioactive molecular entities $[21,22]$. For example, the click strategy is especially versatile for the effective construction of complex glycosylated structures such as clusters, dendrimers, polymers, peptides and macrocycles. In all the cases the triazole ring plays a crucial role in combining divergent units together to establish a complex molecular architecture [23-31]. 
The $\alpha$-GalNAc-linked glycopeptides, $\alpha-N$-glycosidically linked to the polypeptide chain through the amido nitrogen of an asparagine residue at the $N$-terminal [32], were found to be the most important semi-synthetic glycoconjugates, usually modified from their naturally occurring parent precursors [33-39]. Over the years, many structural analogues of this class of antibiotics have been synthesized. In addition, triazoles are considered as peptidic linkage surrogates. Surprisingly, despite the enormous research interests associated with their synthesis, only a few examples of oligosaccharides and glycopeptides mimics have so far been prepared by a click chemistry strategy [40-48]. Most recently, we developed a strategy for the synthesis of 3-amino-2,3-dideoxysugars using a regio- and stereoselective tandem hydroamination/glycosylation of the glycal shown in Figure 1 [49-53]. Extending the synthetic utility of this protocol, herein, we wish to report the synthetic modification of $\alpha$-GalNAc-linked glycopeptides to 3-tosylamino-2,3-dideoxyneoglycoconjugates via click conjugations (Figure 2).

Given the success in using "click chemistry" in the glycosylation reactions, we aspired to apply the highly efficient triazole formation employing an azide $\mathbf{3}$ and a suitable alkyne appended to the 3-amino-2,3-dideoxysugars moiety 2 (Figure 3 ). In

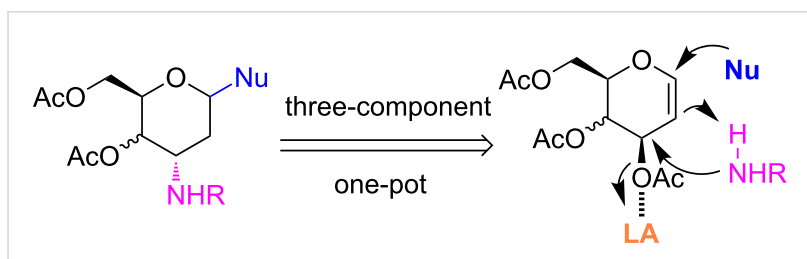

Figure 1: Our reported strategy for quick access to 3-amino-2,3dideoxysugars via regio- and stereoselective tandem hydroamination/ glycosylation of glycals.

continuation of our previous work, herein we report a direct and reliable synthetic approach to multivalent 3-tosylamino-2,3dideoxyneoglyco conjugates 4 with potential biochemical applications involving click conjugations as the key reaction step (Figure 3).

\section{Results and Discussion}

Primarily, we successfully synthesized propargyl 3-p-toluenesulfonamido-4,6-di- $O$-acetyl-2,3-dideoxy- $\alpha$-D-allohexopyranoside (2a) in gram scale via $\mathrm{BF}_{3} \cdot \mathrm{OEt}_{2}$-promoted one-pot threecomponent $\alpha$-selective tandem hydroamination/glycosylation reaction (Scheme 1). In fact, when 3,4,6-tri- $O$-acetyl-D-glucal (1a), propargyl alcohol and $p$-toluenesulfonamide were
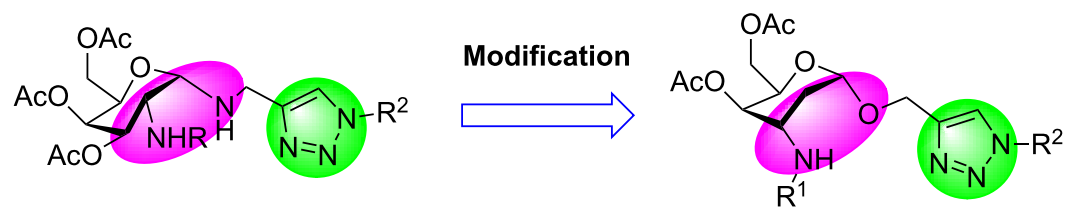

Figure 2: Synthetic modification of a-GalNAc linked glycopeptides to 3-tosylamino-2,3-dideoxyneoglycoconjugates via click conjugation.

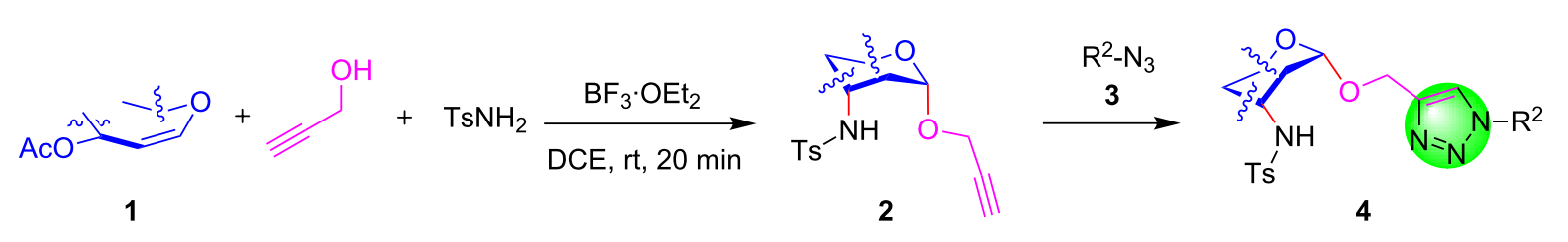

Figure 3: Our proposal for access to 3-tosylamino-2,3-dideoxyneoglycoconjugates via tandem hydroamination/glycosylation of glycals followed by click conjugations.

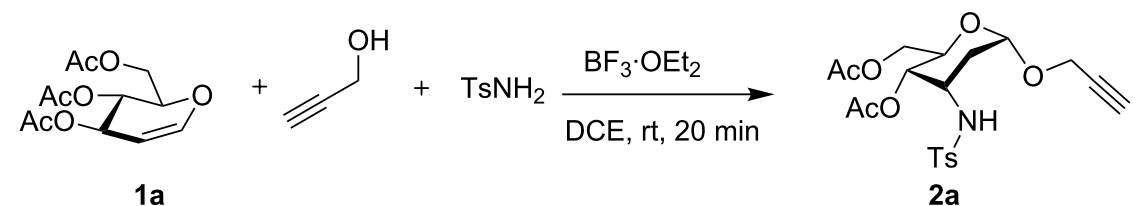

Scheme 1: Synthesis of propargyl 3-tosylamino-2,3-dideoxy-a-D-allohexopyranoside (2a). 
subjected to a one-pot reaction in the presence of 2.2 equiv of $\mathrm{BF}_{3} \cdot \mathrm{OEt}_{2}$ in DCE at room temperature for $20 \mathrm{~min}$, the desired aminoglycoside $\mathbf{2 a}$ was obtained in good yield with exclusive $\alpha$-stereoselectivity [50]. Later, a systematic screening was executed using 3-tosylamino-2,3-dideoxysugar 2a and benzyl azide (3a) as our model system under varied conditions of catalysts, additives, solvents and reaction temperatures (Table 1). The initial evaluation involved no catalyst and additives at $100{ }^{\circ} \mathrm{C}$ and $\mathrm{DMF}, \mathrm{MeCN} / \mathrm{H}_{2} \mathrm{O}$ 3:1 or $\mathrm{MeOH}$ as the solvent system, which resulted in unsuccessful reactions (Table 1, entries 1-3). However, a trace amount of the desired product was detected in the presence of $10 \mathrm{~mol} \%$ of copper(I) iodide (Table 1, entry 4). The combination of $\mathrm{CuSO}_{4} \cdot 5 \mathrm{H}_{2} \mathrm{O}$ $(10 \mathrm{~mol} \%)$ and sodium ascorbate $(10 \mathrm{~mol} \%)$ was found to be a suitable catalyst leading regiospecifically to the 1,4-disubstituted-1,2,3-triazole $4 \mathbf{a}$ with moderate yield of $46 \%$ in $t$-BuOH/ $\mathrm{H}_{2} \mathrm{O} \mathrm{1:1}$ after 20 hours at $70{ }^{\circ} \mathrm{C}$ (Table 1, entry 5). The yield was further improved to $97 \%$ by employing DMF as solvent in a shorter period of 12 hours (Table 1, entry 6). Encouraged by these results, we attempted to improve the assemblies and to shorten the reaction times further; reactions were subjected to microwave irradiation, which is best known to accelerate transition metal-catalyzed homogeneous reactions [54]. Microwaveassisted organic reactions are rapidly becoming recognized as a valuable tool for facilitating a wide variety of organic transformations $[55,56]$. Finally, we found that the rate of conversion accelerated dramatically when microwave irradiation was used under $70{ }^{\circ} \mathrm{C}$. To our delight under microwave conditions and in DMF with addition of $1 \mathrm{~mol} \%$ of $\mathrm{CuSO}_{4} \cdot 5 \mathrm{H}_{2} \mathrm{O}$ and $10 \mathrm{~mol} \%$ of sodium ascorbate, a quantative yield of desired 3-tosylamino2,3-dideoxyneoglycoconjugate 4a was obtained in $15 \mathrm{~min}$ (Table 1, entry 7).
Next, the required $\alpha$-propargyl 3-tosylamino-2,3-dideoxyglycosides 2 were synthesized by $\mathrm{BF}_{3} \cdot \mathrm{OEt}_{2}$-promoted one-pot threecomponent tandem hydroamination/glycosylation reaction on a glycal scaffold including tri- $O$-acetyl-D-glucal (1a), tri- $O$ acetyl-D-allal (1b), tri- $O$-acetyl-D-galactal (1c), di- $O$-acetyl-Drhamnal (1d), hexa- $O$-acetyl-D-maltal (1e). Accordingly, a series of $\alpha$-propargyl 3-tosylamino-2,3-dideoxyglycosides 2a-2d were obtained exclusively with $\alpha$-stereoselectivity in good yields (Table 2, entries 1-5).

With pure $\alpha$-propargyl 3-tosylamino-2,3-dideoxyglycosides and the optimized conditions in hand, we focused on performing a Huisgen cycloaddition reaction. The scope and generality of this method to prepare 3-tosylamino-2,3-dideoxyneoglycoconjugates 4 with the assistance of copper sulfate and sodium ascorbate was examined extensively. A range of $\alpha$-alkyne-3tosylamino-2,3-dideoxysugars and azides with various substituent groups $\left(\mathrm{R}^{2}\right)$ were screened and the summarized results are shown in Table 3. Overall, the yields obtained were from good to excellent while preserving the anomeric selectivity and regioselectivity. In general, the analogous reaction of a set of azides with different substituent groups (3a-3e) with $\alpha$-propargyl 3-tosylamino-2,3-dideoxy glycosides 2 afforded the corresponding 3-tosylamino-2,3-dideoxyneoglycoconjugates $(\mathbf{4 a}-\mathbf{4 h})$ in good to excellent yields with exclusive anomeric selectivity (Table 3, entries 1-8). This encouraging result prompted us to apply these conditions to alkyne $\mathbf{2 a}$ and a series of azido-linked monosaccharides $\mathbf{3 f}, \mathbf{3} \mathbf{g}$ and $\mathbf{3 h}$ as well as the propargyl disaccharide $\mathbf{2 d}$ with $\alpha$-GlaNAc azido $\mathbf{3 g}$ which were also obtained in good yields and selectivities (Table 3, entries 9-13). Subsequently, to shorten the reaction times, we subjected all the click conjugations to microwave irradiation. All the reac-

Table 1: Optimization for synthesis of 3-tosylamino-2,3-dideoxyneoglycoconjugate $\mathbf{4 a}$.

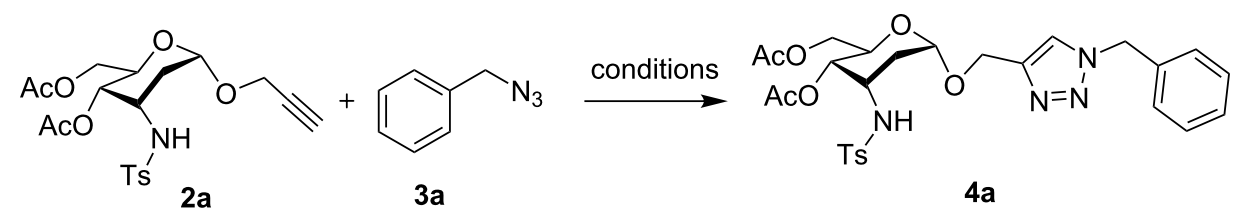

\begin{tabular}{|c|c|c|c|c|c|}
\hline Entry & Catalyst (mol \%) & Solvent & Temperature $\left({ }^{\circ} \mathrm{C}\right)$ & Time (h) & Yield $(\mathrm{mol} \%)^{\mathrm{a}}$ \\
\hline 1 & none & DMF & 100 & 20 & $N R^{b}$ \\
\hline 2 & none & $\mathrm{MeCN} / \mathrm{H}_{2} \mathrm{O}$ & 100 & 20 & $N R^{b}$ \\
\hline 3 & none & $\mathrm{MeOH}$ & 100 & 20 & $N R^{b}$ \\
\hline 4 & Cul (10) & THF & 60 & 12 & trace \\
\hline 5 & $\mathrm{CuSO}_{4} \cdot 5 \mathrm{H}_{2} \mathrm{O}(1)$ & $t-\mathrm{BuOH} / \mathrm{H}_{2} \mathrm{O}$ & 70 & 20 & 46 \\
\hline 6 & $\mathrm{CuSO}_{4} \cdot 5 \mathrm{H}_{2} \mathrm{O}(1)$ & DMF & 70 & 12 & 97 \\
\hline 7 & $\mathrm{CuSO}_{4} \cdot 5 \mathrm{H}_{2} \mathrm{O}(1)$ & DMF & $70^{c}$ & 0.25 & 98 \\
\hline
\end{tabular}

alsolated yield after purification. ${ }^{\mathrm{b}} \mathrm{NR}=$ no reaction. ${ }^{\mathrm{c} A s s i s t e d}$ by microwave irradiation, $200 \mathrm{~W}$. 
Table 2: One-pot synthesis of a-propargyl 3-tosylamino-2,3-dideoxyglycosides 2.

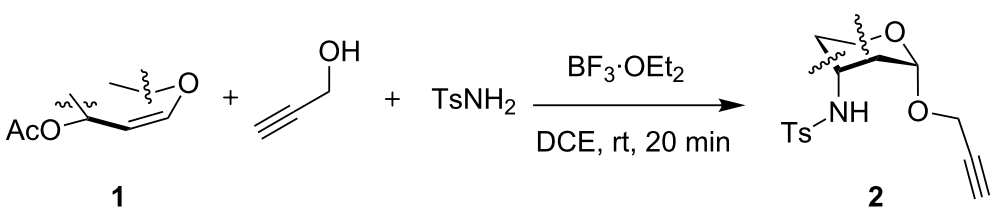

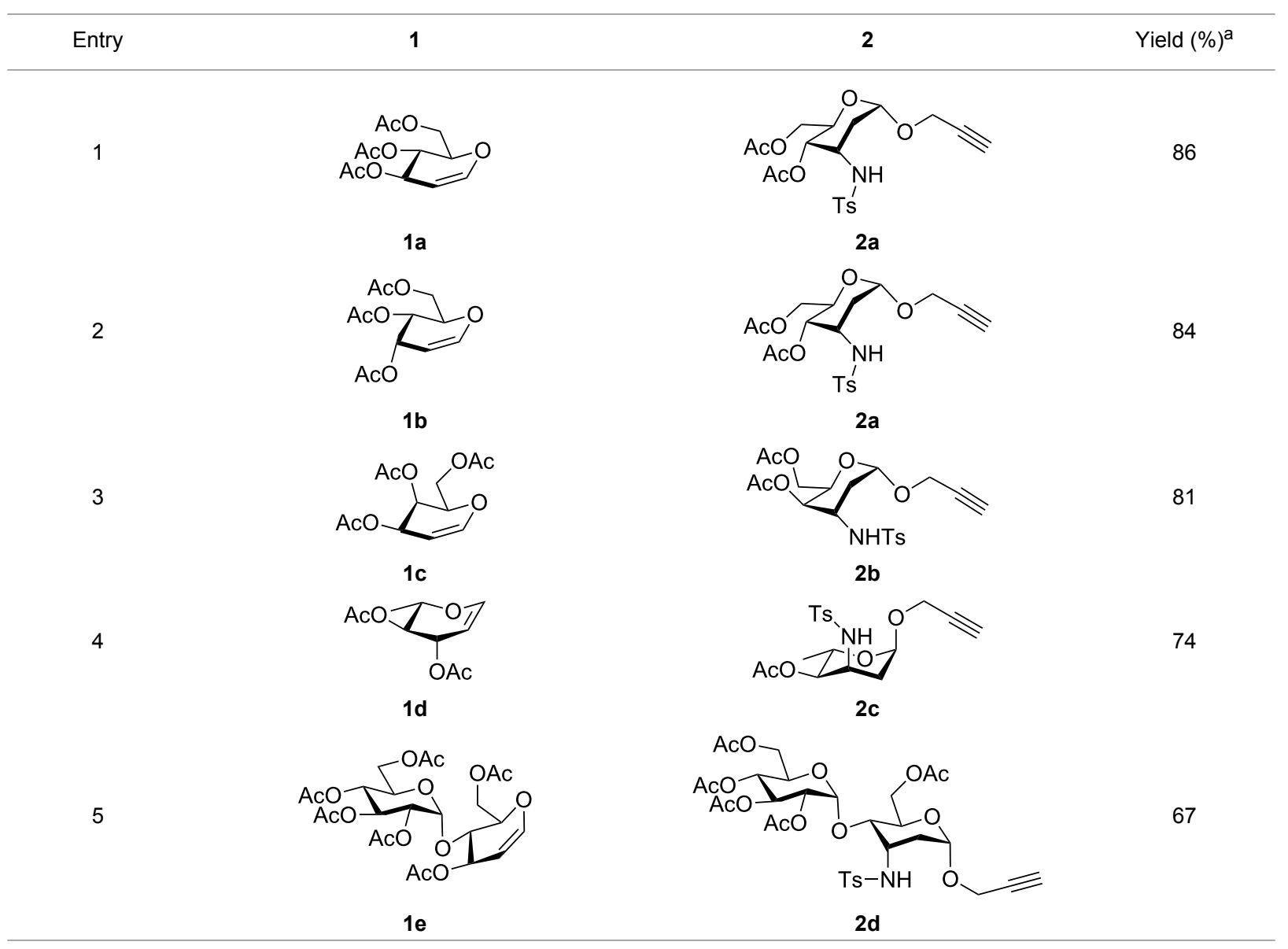

alsolated yields after purification.

tions were completed in considerably shorter reaction times of less than $30 \mathrm{~min}$ for the Huisgen cycloaddition of alkenes and azides catalyzed by copper sulfate and sodium ascorbate, affording the corresponding products in good to excellent yields in each case (Table 3, method B). This result showed that the synthesis of 3-tosylamino-2,3-dideoxyneoglycoconjugates via copper-catalyzed Huisgen cycloaddition is highly efficient under microwave irradiation.

In carbohydrate recognition events, higher multivalent interactions are absolutely essential as the monovalent affinities of carbohydrate monosaccharides are comparatively low and weak. To enhance this multivalent effect, thereby increasing the binding efficiencies of carbohydrates with the coupling counter- parts, there has been a constant development of new glycoconjugates such as glycodendrimers [57]. Hence, as continuation of previous encouraging results, we have further designed the use of noncarbohydrate diazide 5a in the cycloaddition reaction with the $\alpha$-propargyl 3-tosylamino-2,3-dideoxyalloside $\mathbf{2 a}$ and $\alpha$-propargyl 3-tosylamino-2,3,6-trideoxy- $\alpha$-L-ribohexopyranoside 2c (Scheme 2) to obtain divalent 3-tosylamino-2,3dideoxyneoglycoconjugates $\mathbf{6 a}$ and $\mathbf{6 b}$ in $83 \%$ and $61 \%$ yield respectively. The synthesis of trivalent 3-tosylamino-2,3dideoxyneoglycoconjugates $\mathbf{6 c}$ was also feasible by using triazide $\mathbf{5 b}$ in $66 \%$ yield (Scheme 3 ). Interestingly, for all the reactions under microwave irradiation, reaction times were reduced to 15 minutes. As such, this novel synthetic protocol provides a straightforward access to a wide range of 3-tosyl- 
Table 3: Scope for synthesis of 3-tosylamino-2,3-dideoxyneoglycoconjugates.

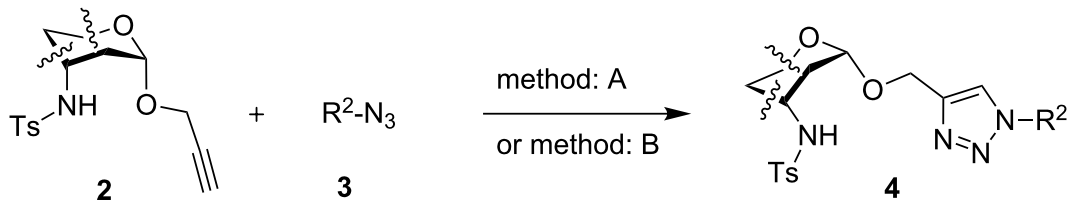

\begin{tabular}{llllll}
\hline Entry & 2 & 3 & 4 & ${\text { Yield }(\%)^{\mathrm{a}}}_{\mathrm{B}^{\mathrm{c}}}^{\mathrm{A}^{\mathrm{b}}}$ \\
\end{tabular}

$12 a$

$3 a$

$2 \quad 2 b$

$3 a$

$32 c$

$4 \quad 2 d$

$3 a$<smiles>[N]C/C=C/c1ccccc1</smiles>

$3 \mathbf{b}$

$6 \quad 2 a$

$\mathrm{N}_{3}$ COOt-Bu

3c

$7 \quad 2 a$<smiles>C=CCC(C)(C)CN</smiles>

$3 d$

$82 a$

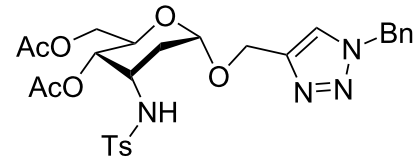

$4 a$

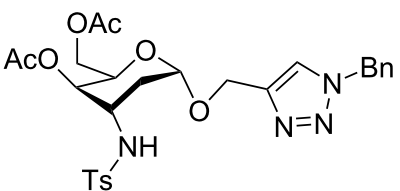

$4 b$

$$
\mathrm{AcO}_{\mathrm{N}}
$$

$74 \quad 81$

4c

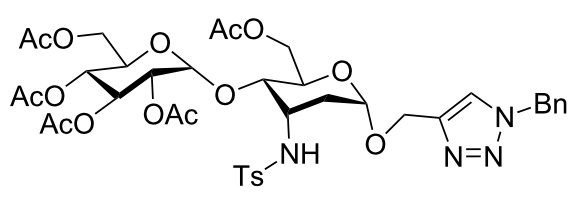

4d

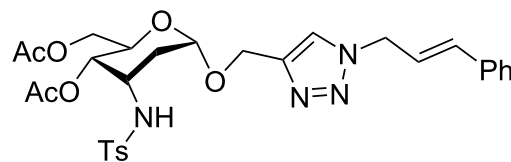

82

85

$4 e$

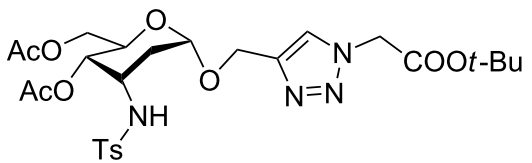

$4 f$

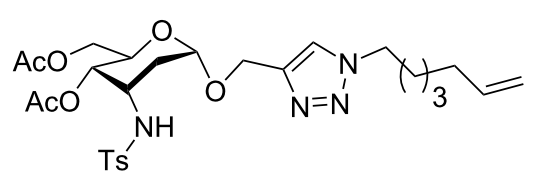

86

89

$4 \mathrm{~g}$

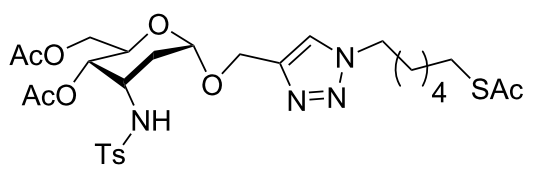

87 92

$4 h$ 
Table 3: Scope for synthesis of 3-tosylamino-2,3-dideoxyneoglycoconjugates. (continued)

$2 a$

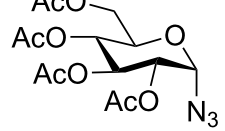

$3 \mathbf{f}$

10

$2 a$

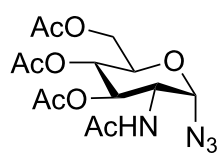

$3 g$

$3 \mathrm{~g}$

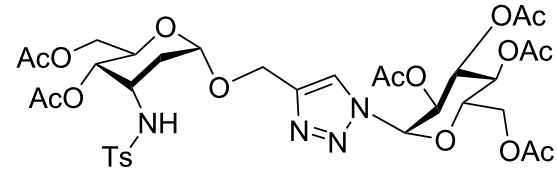

$4 \mathbf{i}$ 4j
11

2d

$2 a$

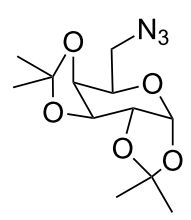

$3 h$

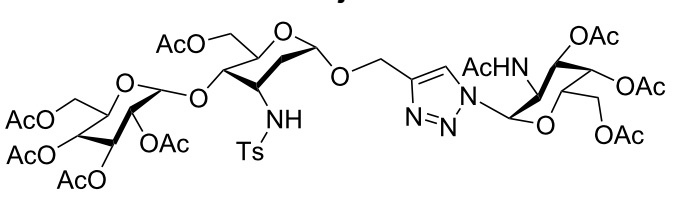

4k

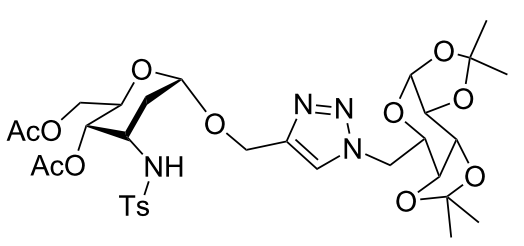

4!

alsolated yields after purification. ${ }^{\mathrm{b}} 70{ }^{\circ} \mathrm{C}$ under conventional heating, 12 hours. ${ }^{\mathrm{C}} 70{ }^{\circ} \mathrm{C}$ under microwave irradiation, $200 \mathrm{~W}, 15 \mathrm{minutes}$.<smiles>[3H]N[C@@H]1C[C@H](OCC#C)O[C@@H](COC(C)(C)C)[C@@H]1OC(C)(C)C</smiles>

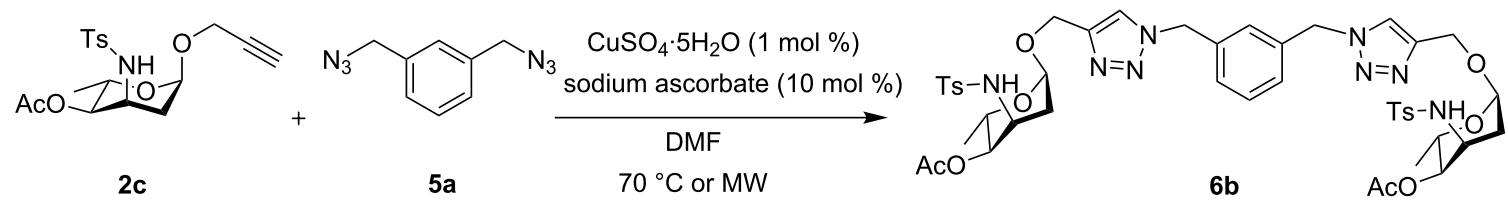

Scheme 2: Synthesis of divalent 3-tosylamino-2,3-dideoxyneoglycoconjugates $\mathbf{6 a}$ and $\mathbf{6 b}$

amino-2,3-dideoxyneoglycoconjugate derivatives which may find numerous biochemical applications [40-48].

\section{Conclusion}

In conclusion, it has been established that the construction of well-defined multivalent, anomerically pure 3-amino-2,3- dideoxyneoglycoconjugate architectures was successfully achieved by using cycloaddition reactions of alkynes and azides. It is expected that this strategy will find extensive applications in glycoscience, because triazole-linked glycoconjugates can exhibit very interesting biological properties, offering a convenient access toward oligosaccharides, glycopeptide 


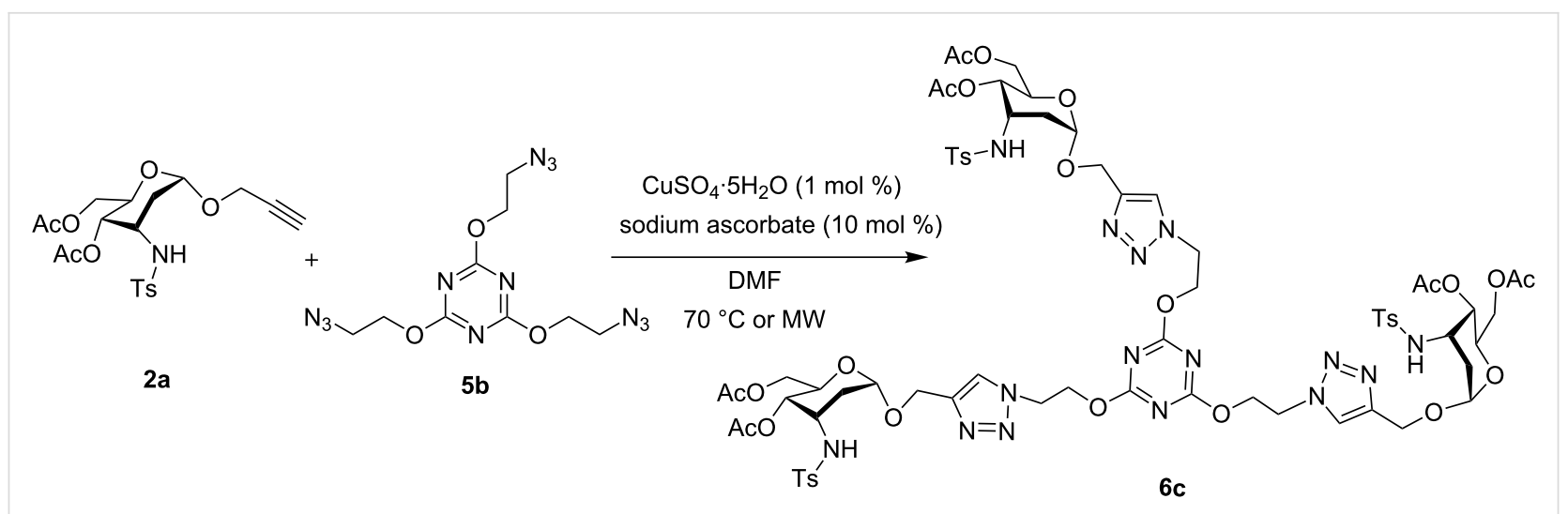

Scheme 3: Synthesis of trivalent 3-tosylamino-2,3-dideoxyneoglycoconjugate 6c.

mimics, or multivalent carbohydrate systems [40-48]. Their further application in molcecular biosystems is currently underway and the results will be reported in due course.

\section{Supporting Information}

\section{Supporting Information File 1}

Experimental, analytical data and ${ }^{1} \mathrm{H}$ NMR and ${ }^{13} \mathrm{C}$ NMR spectra for all new compounds.

[http://www.beilstein-journals.org/bjoc/content/ supplementary/1860-5397-10-134-S1.pdf]

\section{Acknowledgements}

We gratefully acknowledge Nanyang Technological University (RG50/08) and the Ministry of Education, Singapore (MOE 2009-T2-1-030) for the financial support of this research.

\section{References}

1. Driguez, H.; Thiem, J. Glycoscience; Springer: Berlin, 1999; Vol. 1-2.

2. Ernst, B.; Hart, G.; Sinaý, P. Carbohydrates in Chemistry and Biology; Wiley, 2000; Vol. 1-4. doi:10.1002/9783527618255

3. Wang, P. G.; Bertozzi, C. R. Glycochemistry: Principles, Synthesis, and Applications; Marcel Dekker: New York, NY, 2001.

4. Kiessling, L. L.; Gestwicki, J. E.; Strong, L. E. Angew. Chem., Int. Ed. 2006, 45, 2348. doi:10.1002/anie.200502794

5. Gruner, S. A. W.; Locardi, E.; Lohof, E.; Kessler, H. Chem. Rev. 2002, 102, 491. doi:10.1021/cr0004409

6. Huisgen, R. 1,3-Dipolar cycloaddition - Introduction, survey, mechanism. In 1,3-Dipolar Cycloaddition Chemistry; Padwa, A., Ed.; Wiley, 1984; Vol. 1, pp 1-176.

7. Meldal, M.; Tornøe, C. W. Chem. Rev. 2008, 108, 2952. doi:10.1021/cr0783479

8. Rostovtsev, V.; Green, L. G.; Fokin, V. V.; Sharpless, K. B. Angew. Chem., Int. Ed. 2002, 41, 2596. doi:10.1002/1521-3773(20020715)41:14<2596::AID-ANIE2596>3.0.CO $; 2-4$
9. Kolb, H. C.; Finn, M. G.; Sharpless, K. B. Angew. Chem., Int. Ed. 2001, 40, 2004. doi:10.1002/1521-3773(20010601)40:11<2004::AID-ANIE2004>3.0.CO ;2-5

10. Tornøe, C. W.; Christensen, C.; Meldal, M. J. Org. Chem. 2002, 67, 3057. doi:10.1021/j0011148j

11. Davis, B. G. J. Chem. Soc., Perkin Trans. 1 1999, 3215. doi:10.1039/A809773I

12. Turnbull, W. B.; Stoodart, J. F. Rev. Mol. Biotechnol. 2002, 90, 231. doi:10.1016/S1389-0352(01)00062-9

13. Roy, R.; Baek, M.-G. Rev. Mol. Biotechnol. 2002, 90, 291. doi:10.1016/S1389-0352(01)00065-4

14. Bezouška, K. Rev. Mol. Biotechnol. 2002, 90, 269. doi:10.1016/S1389-0352(01)00064-2

15. Dedola, S.; Nepogodiev, S. A.; Field, R. A. Org. Biomol. Chem. 2007, 5, 1006. doi:10.1039/b618048p

16. Dondoni, A. Chem.-Asian J. 2007, 2, 700. doi:10.1002/asia.200700015

17. Patani, G. A.; LaVoie, E. J. Chem. Rev. 1996, 96, 3147. doi:10.1021/cr950066q

18. Tron, G. C.; Pirali, T.; Billington, R. A.; Canonico, P. L.; Sorba, G.; Genazzani, A. A. Med. Res. Rev. 2008, 28, 278. doi:10.1002/med.20107

19. Wilkinson, B. L.; Bornaghi, L. F.; Poulsen, S.-A.; Houston, T. A. Tetrahedron 2006, 62, 8115. doi:10.1016/j.tet.2006.06.001

20. Jung, K.-H.; Schmidt, R. R. Glycosyltransferase Inhibitors. In Carbohydrate-Based Drug Discovery; Wong, C.-H., Ed.; Wiley-VCH Verlag GmbH \& Co KGaA: Weinheim, 2003; pp 609 ff.

21. Kiick, K. L.; Saxon, E.; Tirrell, D. A.; Bertozzi, C. R. Proc. Natl. Acad. Sci. U. S. A. 2002, 99, 19. doi:10.1073/pnas.012583299

22. Jewett, J. C.; Bertozzi, C. R. Chem. Soc. Rev. 2010, 39, 1272. doi:10.1039/b901970g

23. Kolb, H. C.; Sharpless, K. B. Drug Discovery Today 2003, 8, 1128. doi:10.1016/S1359-6446(03)02933-7

24. Whiting, M.; Muldoon, J.; Lin, Y.-C.; Silverman, S. M.; Lindstrom, W.; Olson, A. J.; Kolb, H. C.; Finn, M. G.; Sharpless, K. B.; Elder, J. H.; Fokin, V. V. Angew. Chem., Int. Ed. 2006, 45, 1435. doi:10.1002/anie.200502161

25. Oh, K.; Guan, Z. Chem. Commun. 2006, 3069. doi:10.1039/b606185k 26. Bock, V. D.; Speijer, D.; Hiemstra, H.; van Maarseveen, J. H. Org. Biomol. Chem. 2007, 5, 971. doi:10.1039/b616751a 
27. Angell, Y. L.; Burgess, K. Chem. Soc. Rev. 2007, 36, 1674. doi:10.1039/b701444a

28. Nagarajan, S.; Das, T. M. Carbohydr. Res. 2009, 344, 1028. doi:10.1016/j.carres.2009.03.009

29. Nagarajan, S.; Arjun, P.; Raaman, N.; Das, T. M. Carbohydr. Res. 2010, 345, 1988. doi:10.1016/j.carres.2010.07.016

30. Prasad, V.; Kale, R. R.; Kumar, V.; Tiwari, V. K. Curr. Org. Synth. 2010, 7, 506. doi:10.2174/157017910792246063

31. Pandey, V. P.; Bisht, S. S.; Mishra, M.; Kumar, A.; Siddiqi, M. I.; Verma, A.; Mittal, M.; Sane, S. A.; Gupta, S.; Tripathi, R. P. Eur. J. Med. Chem. 2010, 45, 2381. doi:10.1016/j.ejmech.2010.02.018

32. Shibata, S.; Takeda, T.; Natori, Y. J. Biol. Chem. 1988, 263, 12483.

33. Pajk, S.; Garvas, M.; Štrancar, J.; Pečar, S. Org. Biomol. Chem. 2011, 9, 4150. doi:10.1039/c0ob01173h

34. Kumar, G. D. K.; Baskaran, S. J. Org. Chem. 2005, 70, 4520. doi:10.1021/jo0502697

35. Yan, R.-B.; Yang, F.; Wu, Y.; Zhang, L.-H.; Ye, X.-S. Tetrahedron Lett. 2005, 46, 8993. doi:10.1016/j.tetlet.2005.10.103

36. Alix, A.; Chassaing, S.; Pale, P.; Sommer, J. Tetrahedron 2008, 64, 8922. doi:10.1016/j.tet.2008.06.086

37. Wilkinson, B. L.; Stone, R. S.; Capicciotti, C. J.; Thaysen-Andersen, M.; Matthews, J. M.; Packer, N. H.; Ben, R. N.; Payne, R. J. Angew. Chem., Int. Ed. 2012, 51, 3606. doi:10.1002/anie.201108682

38. Liu, S.; Wang, W.; von Moos, E.; Jackman, J.; Mealing, G.; Monette, R.; Ben, R. N. Biomacromolecules 2007, 8, 1456. doi:10.1021/bm061044o

39. Bouvet, V. R.; Ben, R. N. In Antifreeze Glycoprotein Analogues: Synthesis. In Vitro Testing and Applications; Roy, R., Ed.; American Chemical Society, Oxford University Press: Washington, D.C., 2004; p 151.

40. Santoyo-González, F.; Hernández-Mateo, F. Top. Heterocycl. Chem. 2007, 7, 133. doi:10.1007/7081_2007_050

41. Chen, Q.; Yang, F.; Du, Y. Carbohydr. Res. 2005, 340, 2476. doi:10.1016/j.carres.2005.08.013

42. Gouin, S. G.; Bultel, L.; Falentin, C.; Kovensky, J. Eur. J. Org. Chem. 2007, 1160. doi:10.1002/ejoc.200600814

43. Hotha, S.; Kashyap, S. J. Org. Chem. 2006, 71, 364. doi:10.1021/jo051731q

44. Ortega-Muñoz, M.; Lopez-Jaramillo, J.; Hernandez-Mateo, F.; Santoyo-Gonzalez, F. Adv. Synth. Catal. 2006, 348, 2410. doi:10.1002/adsc.200600254

45. Touaibia, M.; Wellens, A.; Shiao, T. C.; Wang, Q.; Sirois, S.; Bouckaert, J.; Roy, R. ChemMedChem 2007, 2, 1190. doi:10.1002/cmdc.200700063

46. Guo, Z.; Lei, A.; Zhang, Y.; Xu, Q.; Xue, X.; Zhang, F.; Liang, X. Chem. Commun. 2007, 2491. doi:10.1039/b701831b

47. Nepogodiev, S. A.; Dedola, S.; Marmuse, L.; de Oliveira, M. T.; Field, R. A. Carbohydr. Res. 2007, 342, 529. doi:10.1016/j.carres.2006.09.026

48. Pietrzik, N.; Schips, C.; Ziegler, T. Synthesis 2008, 519. doi:10.1055/s-2008-1032150

49. Ding, F.; William, R.; Wang, F.; Ma, J.; Ji, L.; Liu, X.-W. Org. Lett. 2011, 13, 652. doi:10.1021/ol102891t

50. Ding, F. Q.; William, R.; Wang, S.; Gorityala, B. K.; Liu, X.-W. Org. Biomol. Chem. 2011, 9, 3929. doi:10.1039/c1ob05068k

51. Ding, F.; William, R.; Cai, S.; Ma, J.; Liu, X.-W. J. Org. Chem. 2012, 77, 5245. doi:10.1021/jo300791v

52. Ding, F.; William, R.; Liu, X.-W. J. Org. Chem. 2013, 78, 1293. doi:10.1021/jo302619b
53. Ding, F.; Cai, S.; William, R.; Liu, X.-W. RSC Adv. 2013, 3, 13594. doi:10.1039/C3RA40595H

54. Pérez-Balderas, F.; Ortega-Muñoz, M.; Morales-Sanfrutos, J.; Hernández-Mateo, F.; Calvo-Flores, F. G.; Calvo-Asin, J. A.; Isac-García, J.; Santoyo-González, F. Org. Lett. 2003, 5, 1951. doi:10.1021/ol034534r

55. Lidström, P.; Tierney, J.; Wathey, B.; Westman, J. Tetrahedron 2001, 57, 9225. doi:10.1016/S0040-4020(01)00906-1

56. Kappe, C. O.; Pieber, B.; Dallinger, D. Angew. Chem., Int. Ed. 2013, 52, 1088. doi:10.1002/anie.201204103

57. Chabre, Y. M.; Roy, R. Curr. Top. Med. Chem. 2008, 8, 1237. doi:10.2174/156802608785848987

\section{License and Terms}

This is an Open Access article under the terms of the Creative Commons Attribution License

(http://creativecommons.org/licenses/by/2.0), which permits unrestricted use, distribution, and reproduction in any medium, provided the original work is properly cited.

The license is subject to the Beilstein Journal of Organic Chemistry terms and conditions:

(http://www.beilstein-journals.org/bjoc)

The definitive version of this article is the electronic one which can be found at: doi:10.3762/bjoc. 10.134 